\title{
AUSTRALIAN RESEARCH EFFORT FOR THE SKA
}

\author{
WIM BROUW
}

Australia Telescope National Facility, CSIRO, P.O. Box 76, Epping 1710, Australia

\begin{abstract}
The next generation radio telescope being planned is the Square Kilometer Array (SKA): an international project which is currently in the research and development phase. Australia is one of the partner countries in the SKA consortium; here I describe some of the SKA research being undertaken in Australia.
\end{abstract}

Keywords: SKA

\section{Introduction}

The proposed Square Kilometer Array (SKA) is a radio astronomical telescope with the following properties:

- a total reception area of $1 \mathrm{~km}^{2}$

- a total frequency domain of $150 \mathrm{MHz}-20 \mathrm{GHz}$

- high brightness sensitivity (of order $1 \mathrm{~K} @ 1 \operatorname{arcsec}$ at $5 \sigma$ level)

- high spatial resolution $(0.1 \operatorname{arcsec} @ 1.4 \mathrm{GHz})$ and point source sensitivity $\left(A_{\ell f f} / T_{s y s}\right.$ of $2.10^{4} \mathrm{~m}^{2} . \mathrm{K}^{-1}$ )

- a variety of beam forming options, e.g.:

- multiple, simultaneous beams

- fast switching between widely-separated beams, for studies of transient phenomena

- a buffer recording system for studying events discovered by other telescopes

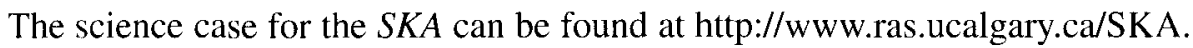

The $S K A$ will be an international project. Possible designs for the telescope and its infrastructure are being researched by groups around the world, represented in an international consortium.

Compared to some of the next generation optical telescopes (e.g. a 100-m telescope), which are being proposed to be built for a comparable budget and time scale, the $S K A$ should be an easier instrument to build. It consists only of the equivalent of $120100-\mathrm{m}$ radio telescopes, 30 years after the first $100-\mathrm{m}$ radio telescope was built. The 100-m optical telescope would be the equivalent of 1208 $\mathrm{m}$ optical telescopes, only a few years after the first monolithic 8-m class telescope was finished. 


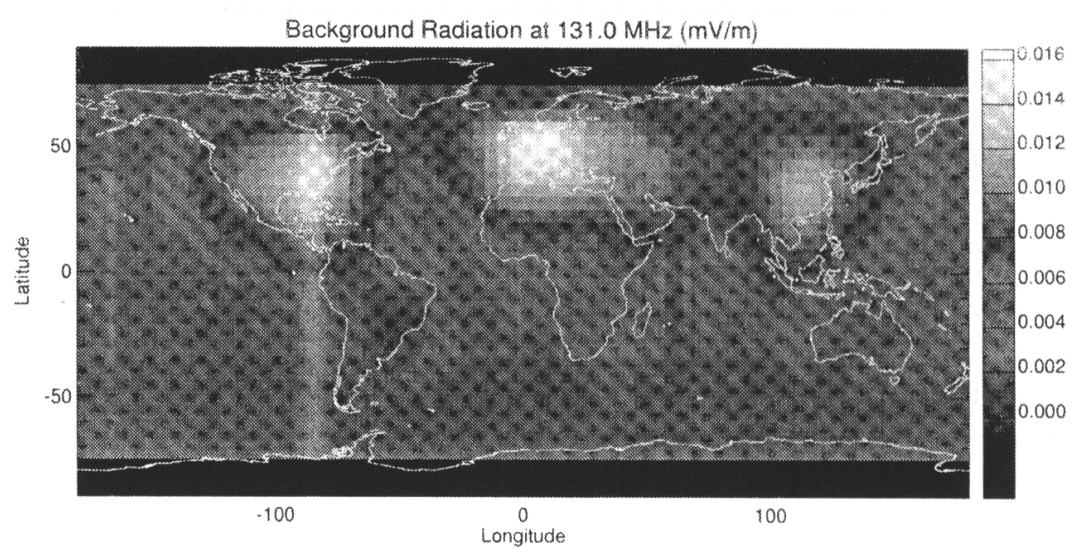

Figure 1. Background radiation at $131 \mathrm{MHz}$, as measured with the Forte satellite.

\section{Australian Research Efforts}

The Australian research for the $S K A$ is concentrated in the following areas:

- international public relations: a brochure has been developed in cooperation with the other members of the $S K A$ consortium

- photonic research, especially in the area of non-linear devices like mixers, filters and $\mathrm{A} / \mathrm{D}$ converters

- external interference mitigation* (in cooperation with USA-based groups), especially in the areas of:

- development of a legal framework for radio-quiet zones

- notch filters

- adaptive nulling

- reference subtraction

- post-correlation detection and correction algorithms

- software radio research (for which a CD with base-band data has been produced that can be used by any group interested in working with real radio astronomical data). Information is available in the proceedings of a December 1999 workshop at http: //www.atnf.csiro.au/SKA.

- optimal configuration studies of the individual elements (an international cooperative program). See e.g. the February 2000 workshop report at the above WWW address.

- site selection issues (in cooperation with governmental representatives), especially:

- legal issues involving historic, cultural, religious, mining and other areas of current and previous use

- measurement issues: how to classify site-related data like interference levels

* Man-made interference is blanking-out large parts of the radio sky, as can be seen in Figure 1. 


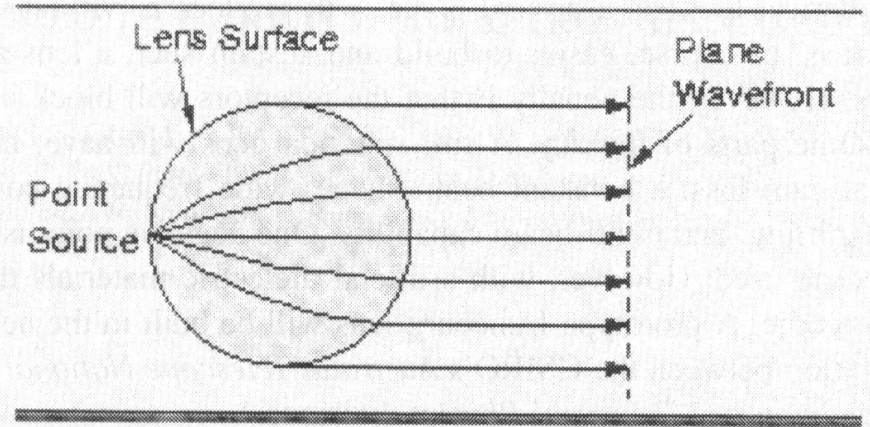

Figure 2. A full spherical Luneburg lens.

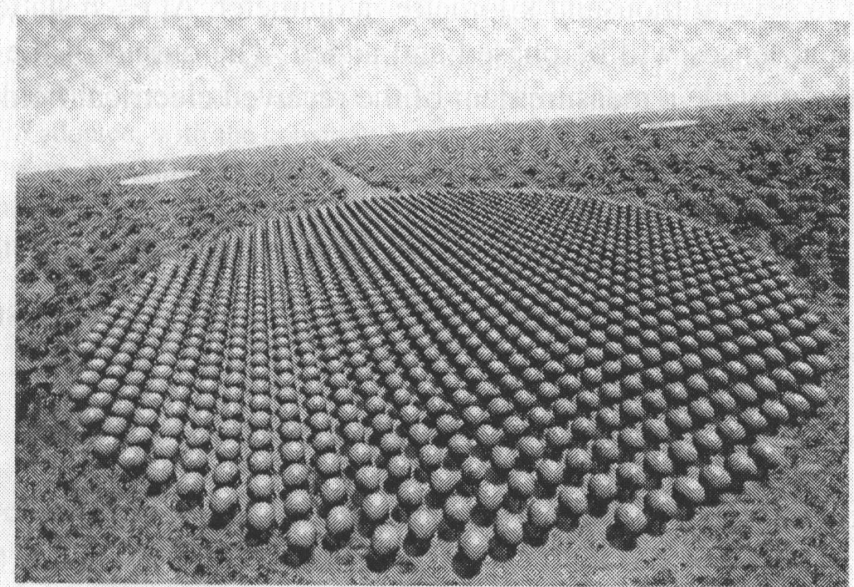

Figure 3. A possible SKA station, based on small Luneburg lenses.

- actual measurements and legal tests for a variety of test sites

- analog concentrators. At the moment a Luneburg lens (see next section) is being studied as a possible analog concentrator.

\section{Luneburg Lens}

The Luneburg lens (James et al., 2000) is a spherical lens which will focus a plane wave from any direction in a point on the opposite side of the lens (see Figure 2). This behaviour is possible if the permittivity, $\epsilon_{r}$, varies as a function of the normalized radius $r$ :

$$
\epsilon_{r}=2-r^{2}
$$

By altering this profile, the focal point can be moved slightly inwards or outwards from the surface. 
Half a spherical lens, on a ground-plane, will produce a 'virtual source' Luneburg lens. It is, of course, easier to build and sustain such a lens as compared to a full lens. However, the penalty is that the receptors will block incident radiation from some parts of the sky. A full spherical lens will have: no blind spot or directional gain loss; a constant beam shape; wide frequency coverage; time delay phase shifting; and multi-beam capability. One negative point is the loss due to the di-electric used. However, with artificial dielectric materials this difficulty could be overcome. A prototype Luneburg lens will be built in the next two years in a collaboration between the CSIRO's Australia Telescope National Facility and Telecommunication and Industrial Physics divisions.

The complete $S K A$ will consist of a number of stations (each of equivalent sensitivity of the order of a $100 \mathrm{~m}$ telescope). These stations will be spread over an area of up to several thousand kilometre in diameter. At each station the output of the individual lenses will be phased and added. The output of the stations will be correlated to provide a measurement of the received electrical field distribution.

\section{References}

James, G., Kot, J., Parfitt, A. and Nikolic, N.: 2000, Luneburg Lens element for the SKA. Proc. SPIE 1015, 410-419. 